\title{
Eventos adversos observados después del tratamiento con factor de transferencia
}

\author{
Adverse events after treatment with the transfer factor
}

\author{
MsC. María Aida Cruz Barrios,' Lic. Blanca Nieves Rodríguez Montiel," \\ MsC. J uan Antonio Furones Mourelle,"' MsC. I smary Alfonso Orta,'" \\ Dra. Deborah Rodríguez Piñerov \\ ' Grupo Empresarial QUI MEFA. La Habana, Cuba. \\ "Facultad de Medicina "Miguel Enríquez". La Habana, Cuba. \\ II' Escuela Nacional de Salud Pública. La Habana, Cuba. \\ IV Unidad Nacional Coordinadora de Farmacovigilancia, MI NSAP. La Habana, Cuba. \\ ${ }^{\vee}$ Grupo Provincial de Farmacoepidemiología. La Habana, Cuba.
}

\section{RESUMEN}

Introducción: el factor de transferencia es un inmunoestimulante que se emplea en una amplia gama de enfermedades. Su eficacia y seguridad han sido evaluadas en ensayos clínicos prerregistro, pero se conoce poco sobre su seguridad en condiciones de la práctica habitual, de ahí la necesidad de vigilar su uso y contribuir a establecer su relación beneficio/riesgo.

Objetivos: identificar eventos adversos en pacientes tratados con factor de transferencia, determinar grado de asociación fármaco-evento observado y su gravedad.

Métodos: estudio observacional, descriptivo, prospectivo y multicéntrico de farmacovigilancia activa. Se observó durante el año siguiente al término del tratamiento con factor de transferencia, a una serie de 282 pacientes tratados por su médico de asistencia entre abril de 2001 y abril de 2002, en 9 hospitales en La Habana. Se analizaron los eventos adversos presentados, su gravedad, si existía asociación con el fármaco, la cantidad de eventos por paciente, la dosis administrada y la edad del paciente. 
Resultados: el $13,8 \%$ de los pacientes observados tuvo al menos un evento adverso, de ellos 38,5 \% tenía entre 45-59 años. Los esquemas de tratamiento con dosis altas y prolongadas no provocaron más eventos que los ya encontrados. Se identificaron 80 eventos adversos, 55 \% leves y en $80 \%$ se consideró improbable su relación con el medicamento. Ninguno se clasificó definitivo o probable, mientras que eventos como fiebre, artralgia, disnea, mialgia, alergia y astenia, se consideraron reacciones adversas posibles y leves.

Conclusiones: la mayoría de los eventos adversos presentados, durante el año siguiente de finalizado el tratamiento con factor de transferencia, fueron leves y no relacionados con su uso. Aun así, los productores del medicamento deberían advertir a profesionales y pacientes sobre las reacciones adversas posibles detectadas.

Palabras clave: Factor de transferencia, reacciones adversas, eventos adversos, efectos adversos, farmacovigilancia, farmacoepidemiología.

\section{ABSTRACT}

Introduction: the transfer factor is an immunologic stimulant used in a wide range of diseases. Its safety and efficiency have been evaluated in pre-registration clinical trials, but little is known about its safety margin under regular practical conditions, hence the need of closely watching its use and setting the benefit-risk relation. Objectives: to identify adverse events in patients treated with the transfer factor and to determine the level of association of the drug and the observed adverse event and severity.

Methods: prospective, multicentered, descriptive and observational study of active drug surveillance. Two hundred and eighty two patients, who were attended by their physicians from April 2001 to April 2002 in 9 hospitals of Havana, were observed for one year after the end of a treatment with the transfer factor. The occurrence of adverse events as well as their severity, association with the drug, the number of events per patient, the supplied dosage and the age of the patient was all analyzed.

Results: in this group of patients, $13.8 \%$ had at least one adverse effect; $38.5 \%$ were aged 45 to 59 years. The treatment schemes with high and prolonged dosage did not cause any further event. Eighty adverse effects were identified, $55 \%$ mild and $80 \%$ were considered as unlikely related with the drug. None of them was definitive or probable whereas fever, arthralgia, dysnea, myalgia, allergy and asthenia were considered as possible mild adverse reactions.

Conclusions: most of adverse events during the year after the end of treatment with the transfer factor were mild and unrelated to the use of the drug. However, the drug manufacturers should advise professionals and patients on the possible occurrence of adverse reactions.

Key words: Transfer factor, adverse reactions, adverse events, adverse effects, drug surveillance, drug epidemiology. 


\section{NTRODUCCIÓN}

La farmacoepidemiología es una rama de la salud pública esencial para cualquier sistema sanitario, porque permite valorar el efecto de los fármacos sobre la morbilidad y la mortalidad en una población definida. ${ }^{1}$ Las investigaciones farmacoepidemiológicas se desarrollan después que los medicamentos se registran para su comercialización, pues existen diferencias entre los resultados de los ensayos clínicos previos al registro y los que se observan después en las condiciones habituales de la práctica médica, básicamente porque un mayor número de personas se exponen al fármaco, sin las condiciones especiales que se dan en estos ensayos y durante un mayor tiempo. ${ }^{1}$

La farmacovigilancia es una de las estrategias principales de la farmacoepidemiología, se ocupa de identificar y valorar las consecuencias del uso, agudo y crónico, de los medicamentos en la población o en subgrupos de pacientes, pero sobre todo en los efectos adversos que provocan, por lo que brinda la posibilidad a las autoridades sanitarias de tomar decisiones sobre su utilización. El método más empleado para detectar efectos adversos es la notificación espontánea que realizan los profesionales sanitarios cuando las sospechan, pero para evitar la infranotificación propia de este método pasivo, se emplean otros como la vigilancia intensiva de pacientes tratados con medicamentos. ${ }^{1,2}$

En Cuba la farmacovigilancia es dirigida por la Unidad Nacional Coordinadora de Farmacovigilancia (UNCF) y se apoya en la red nacional de farmacoepidemiología, que tiene representantes (farmacoepidemiólogos) a nivel provincial, municipal, así como en cada hospital del país. ${ }^{1}$

El factor de transferencia (FT) es un hemoderivado que se emplea en el tratamiento de la inmunodeficiencia celular, consiste en un extracto dializable de leucocitos que transfiere inmunidad de un donante inmune a otro con déficit inmunológico. Se comercializa con el nombre de Hebertrans ${ }^{\circledR}$ y se produce en el Centro de Ingeniería Genética y Biotecnología en La Habana, Cuba. El esquema de tratamiento recomendado es de 1-2 unidades por vía intramuscular o subcutánea, diarias o en días alternos, durante períodos que varían de 1-2 semanas hasta varios meses. El efecto adverso observado en los ensayos clínicos prerregistro es un ligero eritema en el sitio de inyección en el $2 \%$ de los casos. ${ }^{3,4}$

Apenas existen estudios publicados que describan o evalúen los efectos adversos del FT en las condiciones de la práctica médica habitual, así como cualquier evento que pudiera aparecer tras su empleo. Foschi y otros informan lesión cerebral aguda reversible, debilidad, cefalea, confusión mental y hemiparesia en un paciente tratado con FT. ${ }^{5}$ Por su parte, en la UNCF existen escasos reportes de sospecha de reacciones adversas a este medicamento. Estos datos demuestran la necesidad de vigilar el uso del FT, para contribuir a establecer su relación beneficio/riesgo.

El presente trabajo forma parte de una investigación que analiza cómo se prescribe el FT, la evolución clínica de los pacientes tratados y los eventos adversos que se presentaron durante el tratamiento. ${ }^{6-8}$ Los objetivos de esta etapa fueron identificar eventos adversos presentados durante el año siguiente de finalizado el tratamiento con FT, determinar si existe relación entre el fármaco y los eventos observados así como su gravedad. 


\section{MÉTODOS}

Se realizó un estudio observacional, descriptivo, prospectivo y multicéntrico. Dentro de los métodos de farmacovigilancia se empleó el método de vigilancia intensiva. ${ }^{2}$

El diseño contempló la inclusión de todos los pacientes que recibieron tratamiento con FT entre abril de 2001 y abril de 2002, en la consulta externa o en salas de ingreso de 9 hospitales en La Habana: cuatro hospitales clinicoquirúrgicos, dos hospitales pediátricos y tres institutos del tercer nivel de atención sanitaria. ${ }^{8}$

La vigilancia duró todo el año siguiente al último ciclo de tratamiento con FT, debido a los diversos esquemas de tratamientos prescritos, ${ }^{6}$ comenzó en el mes de mayo de 2002 y terminó en mayo de 2004.

En modelos confeccionados al efecto se registraron todos los eventos que se presentaron y no solo la sospecha de reacción adversa al FT, entendiéndose por evento cualquier acontecimiento clínico o de laboratorio que se presentara en el paciente después del tratamiento, se pensara o no que estuviera relacionado con el fármaco. ${ }^{2}$ Otras variables analizadas fueron grado de asociación del evento con el FT, su gravedad, cantidad de eventos por paciente, edad y esquema de tratamiento recibido (unidades totales de FT administradas y duración del tratamiento).

La información fue recogida por el médico prescriptor de FT en consultas mensuales a sus pacientes, bajo la supervisión del farmacoepidemiólogo hospitalario; los datos de los que recibieron el medicamento en la sala del hospital y egresaron, así como de los que no acudieron a la consulta, se obtuvieron por el farmacoepidemiólogo municipal en el hogar del paciente.

Los eventos detectados se analizaron por los autores y un comité de expertos, integrado por especialistas de la UNCF, para valorar su grado de asociación con el FT y gravedad, se tuvieron en cuenta las categorías empleadas en The Uppsala Monitoring Centre ${ }^{9}$ y los criterios de Naranjo, ${ }^{10}$ respectivamente.

Para el análisis de los datos se utilizó la mediana para la variable edad y valores absolutos y relativos para otras variables empleadas.

\section{RESULTADOS}

Se vigilaron 387 pacientes pero en 105 la información no fue adecuada, pues se omitieron datos de medicamentos consumidos por pacientes con enfermedades crónicas, fecha de inicio o término de otros medicamentos consumidos, dosis que recibían, inasistencia a reconsultas y no ser localizados en sus domicilios; en la mayoría de estos casos el paciente o su familia no cooperaron con la recogida de la información. De ahí que se presentan los resultados en 282 pacientes.

La mediana de la edad fue de 26 años (DE: 22,35) con un rango que osciló entre 6 meses y 79 años. La tabla 1 muestra que el 37,6 \% del total de pacientes (grupo predominante) tenía edades entre 0 y 14 años y que el $38,5 \%$ del total de pacientes con eventos adversos tenía entre 45 y 59 años. 
Tabla 1. Pacientes según eventos adversos reportados durante el año siguiente al tratamiento con factor de transferencia y grupo de edad

\begin{tabular}{|c|c|c|c|c|c|c|}
\hline \multirow{3}{*}{$\begin{array}{c}\text { Grupo de } \\
\text { edad (años) } \\
0 \text { a } 14\end{array}$} & \multicolumn{2}{|c|}{ Pacientes sin eventos } & \multicolumn{2}{|c|}{ Pacientes con eventos } & \multicolumn{2}{|c|}{ Total } \\
\hline & No. & $\%$ & No & & No. & $\%$ \\
\hline & 96 & 39,6 & 10 & 25,7 & 106 & 37,6 \\
\hline 15 a 29 & 30 & 12,3 & 12 & 30,7 & 42 & 14,9 \\
\hline 30 a 44 & 42 & 17,3 & 0 & 0,0 & 42 & 14,9 \\
\hline 45 a 59 & 45 & 18,5 & 15 & 38,5 & 60 & 21,3 \\
\hline 60 y más & 30 & 12,3 & 2 & 5,1 & 32 & 11,3 \\
\hline Total & 243 & 100 & 39 & 100 & 282 & 100 \\
\hline
\end{tabular}

Se presentaron eventos adversos en 39 pacientes, 13,8 \% del total. En 23 de ellos, $59 \%$, se presentó un solo evento, en 8 casos se observaron 2, 6 pacientes refirieron de 3 a 5 eventos y 2 pacientes presentaron 6 y 12 eventos, respectivamente. Este último caso era un niño de 6 años, portador de leucemia linfoblástica aguda en fase terminal, que tenía vómitos, diarreas, fiebre, hematuria, eritema generalizado, prurito, aumento de transaminasas, cefalea, artralgia, disnea y cianosis, y que finalmente falleció.

El esquema de tratamiento más empleado fue la administración de 6 a 12 unidades de FT durante 2 meses, $41,5 \%$; pero el que más eventos provocó fue la administración de 16 unidades de FT durante 2 meses, 17,9 \%, como se aprecia en la tabla 2.

Tabla 2. Pacientes según eventos adversos registrados durante el año siguiente al tratamiento con factor de transferencia según esquema de tratamiento

\begin{tabular}{|l|r|r|r|r|r|r|}
\hline $\begin{array}{l}\text { Unidades de FT/duración } \\
\text { del tratamiento en meses }\end{array}$ & $\begin{array}{c}\text { Pacientes sin eventos } \\
\text { No. }\end{array}$ & $\begin{array}{c}\text { Pacientes con eventos } \\
\text { No. }\end{array}$ & \multicolumn{2}{|c|}{$\begin{array}{c}\text { Total } \\
\text { No. }\end{array}$} & $\%$ \\
\hline 6 a $20 /$ menos de 1 & 20 & 8,2 & 4 & 10,2 & 24 & 8,5 \\
\hline 4 a $16 / 1$ & 19 & 7,9 & 7 & 17,9 & 26 & 9,2 \\
\hline 6 a $12 / 2$ & 110 & 45,3 & 7 & 17,9 & 117 & 41,5 \\
\hline $16 / 2$ & 41 & 16,9 & 9 & 23,1 & 50 & 17,8 \\
\hline 20 a $24 / 2$ & 3 & 1,2 & 5 & 12,8 & 8 & 2,8 \\
\hline 12 a $20 / 3$ & 9 & 3,7 & 1 & 2,6 & 10 & 3,6 \\
\hline 30 a 48/3 & 8 & 3,3 & 3 & 7,7 & 11 & 3,9 \\
\hline 16 a 30/4-6 & 1 & 0,4 & 1 & 2,6 & 2 & 0,7 \\
\hline 40 a $72 / 4-6$ & 12 & 4,9 & 1 & 2,6 & 13 & 4,6 \\
\hline 96 a $144 / 1$ año & 20 & 8,2 & 1 & 2,6 & 21 & 7,4 \\
\hline Total & 243 & 100 & 39 & 100 & 282 & 100 \\
\hline
\end{tabular}

En general se observó que los esquemas de tratamiento con dosis altas y los prolongados no provocaron más eventos que los ya encontrados.

Se registraron 80 eventos adversos, los más frecuentes fueron muerte de 10 pacientes, diarreas en 8 , fiebre en 6 , cefalea y artralgia en 4 pacientes, así como dolor abdominal y astenia en 3 de ellos. El resto de los eventos se presentó en 1 o 2 pacientes cada uno, como se observa en la tabla 3. 
Al analizar si existía relación con la administración de $\mathrm{FT}$, ningún evento se consideró como una reacción adversa definitiva ni probable, sobre todo porque los pacientes no se reexpusieron al medicamento y existían causas alternativas que pudieran explicar mejor el evento presentado.

Tabla 3. Eventos adversos registrados durante el año siguiente al tratamiento con factor de transferencia según asociación de causalidad

\begin{tabular}{|c|c|c|c|}
\hline Clasificación & Evento adverso (cantidad de pacientes) & No. & $\%$ \\
\hline Posible & $\begin{array}{l}\text { Artralgia (1), disnea (1), fiebre (1), mialgia (1), alergia (1), } \\
\text { astenia (1) }\end{array}$ & 6 & 7,5 \\
\hline Condicional & Astenia (1), esquizofrenia (1), lipotimia (1), neoplasia de mama (1) & 4 & 5,0 \\
\hline Improbable & $\begin{array}{l}\text { Muerte (10), diarrea (8), fiebre (4), cefalea (3), artralgia (3), } \\
\text { dolor abdominal (3), sangramiento (2), cianosis (2), } \\
\text { nasofaringitis (2), anemia (2), disnea (1), astenia (1), aumento } \\
\text { de transaminasas (1), dolor lumbar (1), vómito (1), hipertensión } \\
\text { arterial (1), isquemia transitoria (1), aborto espontáneo (1), } \\
\text { gastritis (1), alopecia (1), eritema generalizado (1), prurito (1), } \\
\text { constipación (1), parestesia (1), edema bucal y palpebral (1), } \\
\text { urticaria (1), hipertensión endocraneana (1), conjuntivitis } \\
\text { bacteriana (1), fractura de un diente (1), convulsión (1), } \\
\text { sudoración (1), cistitis (1), tos (1), náuseas (1), embarazo } \\
\text { ectópico (1). }\end{array}$ & 64 & 80,0 \\
\hline No evaluable & $\begin{array}{l}\text { Fiebre(1), cefalea(1), aumento de transaminasas(1), } \\
\text { varicocele(1), quistes hepáticos(1), trombosis venosa (1) }\end{array}$ & 6 & 7,5 \\
\hline \multicolumn{2}{|l|}{ Total } & 80 & 100 \\
\hline
\end{tabular}

La tabla 3 también muestra que 6 eventos se clasificaron como reacciones adversas posibles, 4 eventos se consideraron reacciones adversas condicionales porque se requerían más datos para su análisis y en 6 eventos observados no se pudo clasificar su asociación con el FT, porque la información resultó contradictoria o insuficiente.

Para la mayoría de los eventos, 80 \%, la asociación con el FT se valoró de improbable, principalmente porque la secuencia temporal entre el inicio del evento y el evento en sí no era favorable. Por ejemplo, los 10 pacientes que fallecieron eran portadores de enfermedades neoplásicas (rabdomiosarcoma cervical, tumor del tallo cerebral, neuroblastoma, sarcoma de Ewing, retinoblastoma, leucemia linfoide aguda) y fallecieron por cuadros característicos de la enfermedad de base, después de 1-2 meses de la exposición al FT.

En la tabla 4 se observa que el $55 \%$ del total de eventos registrados se clasificaron como leves. Las 6 reacciones adversas posibles se incluyeron en esta categoría. 
Tabla 4. Eventos adversos registrados durante el año siguiente al tratamiento con factor de transferencia según su gravedad

\begin{tabular}{|l|r|r|}
\cline { 2 - 2 } \multicolumn{1}{|c|}{ Intensidad } & No. & \% \\
de los eventos adversos & No. & \% \\
\hline Leves & 44 & 55,0 \\
\hline Moderados & 15 & 18,7 \\
\hline Graves & 11 & 13,8 \\
\hline Fatales & 10 & 12,5 \\
\hline Total & 80 & 100 \\
\hline
\end{tabular}

De intensidad moderada, $18,8 \%$, se consideró la esquizofrenia, convulsiones, fiebre, vómitos y diarreas en un paciente; eritema generalizado, prurito, aumento de transaminasas, cefalea, cianosis y artralgia en otro; hipertensión arterial, accidente isquémico transitorio, lipotimia y aborto espontáneo. Mientras que los graves, $13,8 \%$, fueron hipertensión endocraneal, sangramientos, diarreas, dolor abdominal, sudoraciones y cianosis, todos en un caso, sangramientos y disnea, neoplasia de mama, embarazo ectópico y varicocele.

\section{DISCUSIÓN}

Un año después de haber recibido tratamiento con FT, la mayoría de los eventos adversos que se registraron en los pacientes tratados no se consideraron relacionados con su administración y fueron leves. Estos resultados hablan a favor de su seguridad.

Algunos sesgos pudieron estar presentes en esta investigación, como el que los médicos registraran solo aquellos eventos que ellos consideraban como provocados por el FT y no todos los que se presentaran, o el de memoria por parte de los pacientes, teniendo en cuenta que fueron interrogados mensualmente durante un año.

A diferencia de lo que refiere la literatura, ${ }^{2}$ no se observaron más eventos en niños o ancianos que en el adulto. Además los pacientes que utilizaron dosis mayores no presentaron más eventos, sino al contrario, igual resultado se observó durante el tratamiento. ${ }^{8}$ Todo esto puede estar relacionado con la cantidad de pacientes incluidos en el estudio y los sesgos mencionados, de ahí la necesidad de vigilar el uso del FT mientras el medicamento permanezca comercializado, porque para evaluar su relación beneficio-riesgo es necesario disponer de información sobre su uso en las condiciones de la vida real, cuanta más mejor y obtenida con diferentes métodos para así compensar las limitaciones de cada uno de ellos.

Los estudios descriptivos no son los apropiados para determinar la relación temporal entre una posible causa y su efecto, pero razones metodológicas y éticas hacen poco habitual experimentar en farmacovigilancia, ${ }^{2}$ además en el diseño de esta investigación no fue factible comparar el FT con otros inmunoestimulantes. Sin embargo, el método empleado, vigilancia intensiva de los pacientes tratados, fue capaz de generar una señal: el FT posiblemente provoca reacciones adversas; se deberían realizar estudios epidemiológicos más específicos encaminados a 
verificarlas y así conducir o no a la conclusión de que el medicamento causó estos eventos.

Reacciones adversas probables como fiebre, astenia y mialgia, que se observan durante el tratamiento con $\mathrm{FT}^{8}$ volvieron a observarse durante el año siguiente, aunque con una categoría de asociación posible. Otras reacciones adversas potenciales que se identificaron, como artralgia, disnea y alergia, también se observaron durante el tratamiento. ${ }^{8}$

Finalmente puede decirse que a pesar de que las reacciones adversas no fueron frecuentes, durante el año siguiente de finalizado el tratamiento con factor de transferencia, los productores de este fármaco deberían tenerlas en cuenta y advertir en la información que acompaña al medicamento, a profesionales y pacientes, que es posible su aparición con el uso.

\section{REFERENCI AS BI BLI OGRÁFICAS}

1. Furones JA, Pérez J. Necesidad de la Farmacoepidemiología. En: Colectivo de autores, editores. Farmacoepidemiología. Uso racional de medicamentos. La Habana: Editorial Academia; 2010. p. 1-14.

2. Furones JA. Reacciones adversas. Farmacovigilancia e interacciones medicamentosas. En: Vergel Rivera G, Tasé Martínez MJ, Groning Roque E, editores. Farmacología en el proceso de atención de enfermería. La Habana: Editorial Ciencias Médicas; 2009. p. 52-88

3. Fernández CB. Factor de transferencia, nuevas actividades biológicas. Avances Médicos Cuba. 1999; 7:30-2.

4. Simposio Internacional sobre Interferón y Citoquinas. La Habana, Cuba. Diciembre 2-5, 2001[Internet]. [citado 21 diciembre 2007]. Disponible en: http://elfosscientiae.cigb.edu.cu/PDFs/BA/2001/18/Especial/BA00180E013-024.pdf

5. Foschi FG, Marsigli L, Bernardi M, Salvi F, Mascalchi M, Gasbarrini G, et al. Acute multifocal cerebral white matter lesions during transfer factor therapy. J Neurol Neurosurg Psychiatry [Internet]. 2000 [citado Ene 2002];68. Available from: http://jnnp.bmj.com/content/68/1/114. full

6. Cruz MA, Rodríguez BN, Furones JA, Martín AD, Guerra LM, Páez AT. Patrones de prescripción de factor de transferencia en 11 hospitales de Ciudad de La Habana, año 2002. Rev Cubana Salud Pública [Internet]. 2005 [citado 23 Abr 2010]; 31(4). Disponible en: http://bvs.sld.cu/revistas/spu/vol31_4_05/spu04405.htm

7. Cruz MA, Rodríguez BN, Furones JA, Pérez E, Morris AC, Huete S. Evolución clínica de pacientes tratados con factor de transferencia. Rev Cubana Hematología [Internet]. 2004 [citado 23 Abr 2010];20(3). Disponible en: http://bvs.sld.cu/revistas/hih/vol20_3_04/hih06304.htm 
8. Cruz MA, Rodríguez BN, Furones JA, Alonso L, Calvo DM, Rego JJ. Efectos adversos asociados al tratamiento con factor de transferencia. Ciudad de La Habana, 2004. Rev Cubana Farm [Internet]. 2006 [citado 23 Abr 2010];40(1). Disponible

en:

http://bvs.sld.cu/revistas/far/vol40_1_06/far04106.htm

9. Vigilancia de la seguridad de los medicamentos. Guía para la instalación y puesta en funcionamiento de un centro de farmacovigilancia [Internet]. WHO: Uppsala Monitoring Centre [citado 24 May 2002]. Disponible en: http://whoumc.org/graphics/24751.pdf

10. Naranjo CA, Busto UE. Reacciones adversas a medicamentos. En: Naranjo CA, editor. Métodos de farmacología clínica. Washington, D.C.: OPS/OMS; 1992. p. 331.

Recibido: 20 de julio de 2011.

Aprobado: 14 de septiembre de 2011.

María Aida Cruz Barrios. Calle Ramón Pintó No. 202, Luyanó, municipio 10 de Octubre. La Habana, Cuba.

Telef.: 690 6658. Correo electrónico: maria.cruz@infomed.sld.cu 